\title{
EXPERIMENTAL PREDICTIONS FROM TECHNICOLOR THEORIES
}

\author{
S. DIMOPOULOS and S. RABY \\ Physics Department, Stanford Untversity, Stanford, CA 94305, USA \\ G. L. KANE \\ Randall Laboratory of Phystcs, University of Michigan, Ann Arbor, MI 48109, USA
}

Received 6 August 1980

\begin{abstract}
We calculate detailed predictions for masses, decay branching ratıos, and production cross sections for new dynamical bosons expected as physical particles in a technicolor theory The colored technieta state $(m=240 \mathrm{GeV})$ has a significant production cross section at Isabelle or the Tevatron collider. Light, neutral, spın-zero axıons $(m \leqslant 2.5 \mathrm{GeV})$ are expected, and also charged, spın-zero, axions (analogous to charged Higgs bosons) with mass $8 \mathrm{GeV}$ The latter should be seen at PETRA/PEP.
\end{abstract}

\section{Introduction}

The standard model of electroweak [1] and strong interactions is by now well established at low energies. A serious flaw of the standard model is the existence of fundamental scalars [2]. It limits the predictive power of the model by introducing numerous arbitrary parameters. In addition, scalars cannot naturally account for the fact that the electroweak breaking scale $(300 \mathrm{GeV})$ is much less than the cutoff scale $\left(\sim 10^{16} \mathrm{GeV}\right)$.

Recently some attempts have been made to construct theories without fundamental scalars [2-17]. A common feature of these attempts is the existence of a new strong interaction, called technicolor, at the scale of $\sim 1 \mathrm{TeV}$. An immediate consequence of this scenario is the existence of technihadrons (technirho, technisigma, technibaryons, etc.) at the $1 \mathrm{TeV}$ scale.

The purpose of this paper is to discuss some possible consequences of these theories at energies between a few $\mathrm{GeV}$ and several hundred $\mathrm{GeV}$. These consequences are very different from what one expects to see in theories with fundamental scalars. They are associated with the pseudo-Nambu-Goldstone bosons that may occur in such theories [4, 8-14]. These pseudos have masses ranging from a few $\mathrm{GeV}$ to about $250 \mathrm{GeV}$. Consequently they may show up in the next generation of accelerators, or even at present machines.

In sect. 2 we discuss the quantum numbers and masses of these pseudos in a simple model. In sect. 3 we consider their dominant decay modes and evaluate their decay rates. In sect. 4 we compute some interesting production cross sections for these pseudos, and in sect. 5 we discuss the consequences for experiment. Details of mass calculations are in the appendices. 
Our main results are a set of masses and decay widths for technihadrons that are much more detailed and complete than in previous work. Hopefully this will assist in relating the technicolor ideas to experiment. In particular, two results should be emphasized. First, the colored technieta can be produced with a detectable cross section at Isabelle or the Tevatron collider. Second, and most important for now, the masses of the charged axions have been carefully estimated and should be about $8 \mathrm{GeV}$, so there is good reason to hope for their discovery at PETRA or PEP. We also expect light neutral axions ( 2 states) with masses of about $2.5 \mathrm{GeV}$; they will decay dominantly to ss with at least a $10 \%$ branching ratio to $\mu^{+} \mu^{-}$.

\section{Pseudotypes}

In this section we wish to exhibit the quantum numbers and the masses of the pseudos that occur in a large class of models. We shall assume that there exists one complete family of technifermions

$$
\left(\begin{array}{l}
U_{L} \\
D_{L}
\end{array}\right)_{r, y, b}\left(\begin{array}{lll}
N_{L} \\
E_{L}
\end{array}\right) \quad \begin{array}{ll}
U_{R r, y, b} & N_{R} \\
D_{R r, y, b} & E_{R}
\end{array}
$$

where the technifermions have the standard $\mathrm{SU}(3) \times \mathrm{SU}(2)_{\mathrm{L}} \times \mathrm{U}(1)_{Y}$ quantum numbers.

Let us also assume that the technicolor group is $\mathrm{SU}(N)$ and the technifermions belong to the $N$-dimensional representation ${ }^{\star}$.

In the absence of the $\mathrm{SU}(3) \times \mathrm{SU}(2)_{L} \times \mathrm{U}(1)_{Y}$ forces there exists an $\mathrm{SU}(8)_{L} \times$ $\mathrm{SU}(8)_{\mathrm{R}}$ chiral symmetry.

Following the scenario of refs. [2-13] we assume that when the technicolor forces become strong the following condensates form:

$$
\left\langle\overline{\mathrm{U}}_{\mathrm{L}} \mathrm{U}_{\mathrm{R}}\right\rangle_{\mathrm{r}, \mathrm{y}, \mathrm{b}}=\left\langle\overline{\mathrm{D}}_{\mathrm{L}} \mathrm{D}_{\mathrm{R}}\right\rangle_{\mathrm{r}, \mathrm{y}, \mathrm{b}}=\left\langle\overline{\mathrm{N}}_{\mathrm{L}} \mathrm{N}_{\mathrm{R}}\right\rangle=\left\langle\overline{\mathrm{E}}_{\mathrm{L}} \mathrm{E}_{\mathrm{R}}\right\rangle .
$$

These condensates break the $\mathrm{SU}(8)_{\mathrm{L}} \times \mathrm{SU}(8)_{\mathrm{R}}$ chiral symmetry down to $\mathrm{SU}(8)_{\mathrm{L}+\mathrm{R}}$. The $8^{2}-1$ broken generators then produce 63 Nambu-Goldstone bosons. Note that three of these bosons give mass to the $\mathrm{W}^{ \pm}$and $\mathrm{Z}^{0}$ bosons. In table 1 we list these bosons with their $\mathrm{SU}(3) \times \mathrm{SU}(2)_{\mathrm{L}} \times \mathrm{U}(1)_{Y}$ quantum numbers as well as their masses. Their mass is generated by the $\mathrm{SU}(3) \times \mathrm{SU}(2)_{\mathrm{L}} \times \mathrm{U}(1)_{Y}$ forces as well as the extended technicolor and Pati-Salam forces. Detailed calculations of the masses of these bosons have been done in refs. [8, 9, 11-13]. We believe that the contributions of the $S U(3) \times S U(2)_{L} \times U(1)_{Y}$ forces to the masses of these bosons are reliable. In contrast, the contribution of the extended technicolor-Pati-Salam forces are model dependent. As a result the masses of the light bosons are uncertain. We shall, however, estimate the contribution to the mass of the light pseudos coming from Pati-Salam forces at the end of this section.

* Our discussion does not sensitively depend on the assumption that the techni-fermions belong to the $N$-dimensional representation of $\operatorname{SU}(N)$. It is, however, strongly dependent on the assumption of the existence of one and only one complete techni-family 
TABLE 1

Pseudo Nambu-Goldstone bosons of the model

\begin{tabular}{|c|c|c|c|c|}
\hline Pseudo & Color & Charge & Mass $(\mathrm{GeV})$ & Name \\
\hline $\begin{array}{l}\bar{U} U-\bar{D} D+\bar{N} N-\bar{E} E \\
\bar{U} D+\bar{N} E\end{array}$ & $\begin{array}{l}1 \\
1\end{array}$ & $\begin{array}{r}0 \\
-1\end{array}$ & & $\begin{array}{l}\text { Technıpions } \\
\text { (eaten by gauge bosons) }\end{array}$ \\
\hline $\bar{U} \frac{1}{2} \lambda^{a} U+\bar{D} \frac{1}{2} \lambda^{a} D$ & 8 & 0 & & colored technieta $\eta_{\mathrm{T}}^{\mathrm{a}}$ \\
\hline $\bar{U} \frac{1}{2}^{1} \lambda^{a} U-\bar{D} \frac{1}{2} \lambda^{a} D$ & 8 & 0 & $240 \sqrt{\frac{4}{N}}$ & colored technipions \\
\hline $\bar{U} \frac{1}{2} \lambda^{a} D$ & 8 & -1 & & $\pi_{\mathrm{T}}^{\mathrm{a} 3}, \pi_{\mathrm{T}}^{\mathrm{a} \pm}$ \\
\hline $\bar{E} U$ & 3 & $\frac{5}{3}$ & $165 \sqrt{\frac{4}{N}}$ & \\
\hline$\sqrt{\frac{1}{2}}(\bar{E} D-\bar{N} U)$ & 3 & $\frac{2}{3}$ & $160 \sqrt{\frac{4}{N}}$ & technileptoquark \\
\hline $\bar{N} D$ & 3 & $-\frac{1}{3}$ & $155 \sqrt{\frac{4}{N}}$ & \\
\hline$\sqrt{\frac{1}{2}}(\bar{N} U+\bar{E} D)$ & 3 & $+\frac{2}{3}$ & $160 \sqrt{\frac{4}{N}}$ & \\
\hline $\bar{U} U+\bar{D} D-(\bar{N} N+\bar{E} E)$ & 1 & 0 & $\begin{array}{l}\text { see } \\
\text { table } 4\end{array}$ & paraxion $\mathrm{a}_{\mathrm{T}}^{0}$ \\
\hline $\begin{array}{l}\bar{U} U-\bar{D} D+3(\bar{E} E-\bar{N} N) \\
\bar{U} D-3 \bar{N} E\end{array}$ & $\begin{array}{l}1 \\
1\end{array}$ & $\begin{array}{c}0 \\
-1\end{array}$ & & $\begin{array}{l}\text { axion } \mathrm{a}_{\mathrm{T}}^{3} \\
\text { charged axion } \mathrm{a}_{\mathrm{T}}^{ \pm}\end{array}$ \\
\hline
\end{tabular}

Before we do so we must discuss the relevant scales of the problem. The scale of symmetry breaking as measured by the technipion decay constant $F_{\mathrm{T}}$ is determined by the requirement that the $\mathrm{W}^{ \pm}$and $\mathrm{Z}^{0}$ bosons have the right mass.

Let $\psi$ be the column of 4 -component Dirac fields:

$$
\psi=\left\{\begin{array}{c}
U_{r} \\
U_{y} \\
U_{b} \\
N \\
D_{r} \\
D_{y} \\
D_{b} \\
E
\end{array}\right\}
$$

The broken axial generators are defined by

$$
J_{\mu 5}^{a}=\bar{\psi} \gamma_{\mu} \gamma_{5} t^{a} \eta_{\mathrm{T}} \psi,
$$

where $t^{a}$ are the $\mathrm{SU}(8)$ generators normalized by $\operatorname{Tr}\left(t^{a} t^{b}\right)=\frac{1}{2} \delta^{a b}$ and $\nabla_{\mathrm{T}}$ is the unit matrix in technicolor space.

The technipion decay constant is defined by

$$
\left\langle 0\left|J_{\mu 5}^{a}(0)\right| \pi_{\mathrm{T}}^{b}(q)\right\rangle=i F_{\mathrm{T}} q_{\mu} \delta_{a b} .
$$


The conventions are those of Bjorken and Drell except for the normalization of the single-particle states which is taken to be

$$
\left\langle\pi_{\mathrm{T}}^{a}(q) \mid \pi_{\mathrm{T}}^{b}(k)\right\rangle=(2 \pi)^{3} 2 q_{0} \delta^{3}(q-k) \delta_{a b} .
$$

The SU $(2)_{L}$ currents are given by

$$
J_{\mu \mathrm{L}}^{i}=\bar{\psi} \gamma_{\mu}\left(\frac{1-\gamma_{S}}{2}\right) \frac{\tau^{\imath}}{2} \mathbb{\pi}_{\mathrm{T}} \psi
$$

where

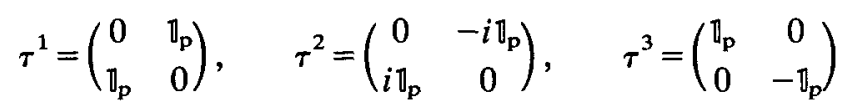

and $\mathbb{v}_{\mathrm{p}}$ is a $4 \times 4$ unit Pati-Salam matrix. Note that $\operatorname{Tr}\left(\frac{1}{2} \tau^{2} \frac{1}{2} \tau^{\prime}\right)=\frac{1}{2} 4 \delta_{l v}$.

The hypercharge current is

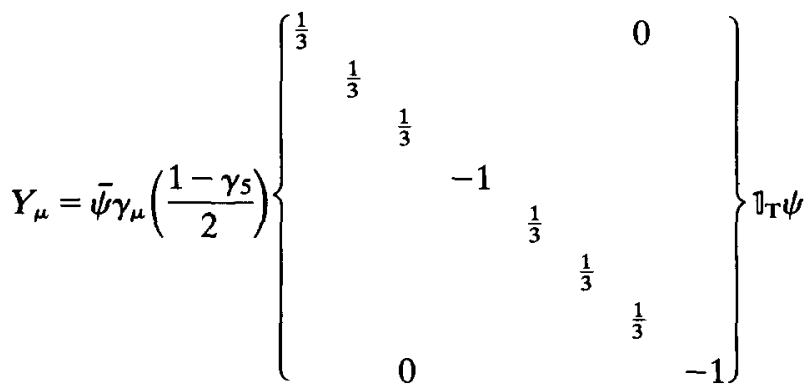

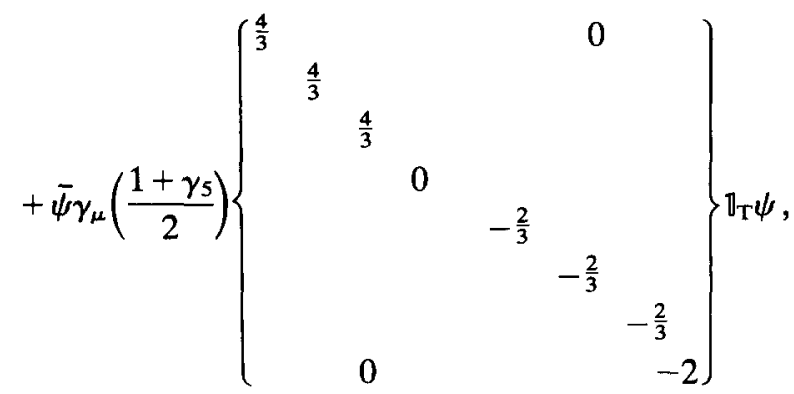

or

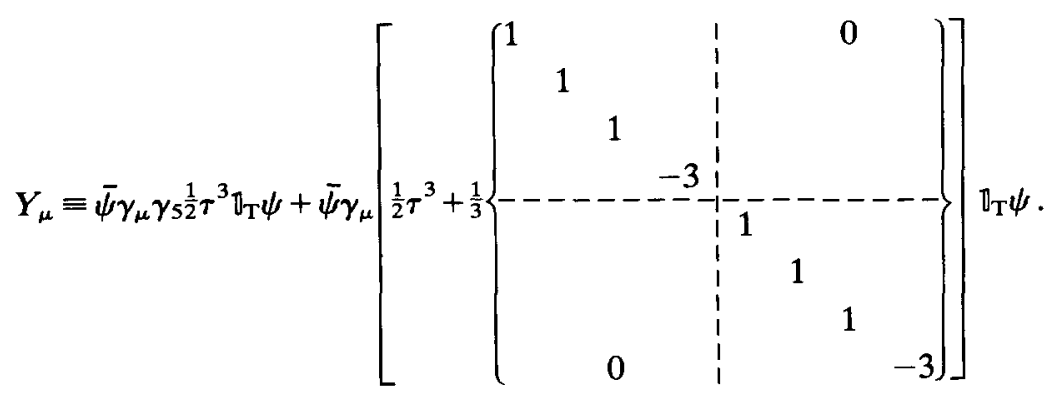


With these definitions in hand let us compute the electroweak vector boson mass matrix. As a result we shall obtain the value of $F_{\mathrm{T}}$ in this model.

Consider the axial current

$$
J_{\mu 5}^{l}=\bar{\psi} \gamma_{\mu} \gamma_{5} \frac{\tau^{\prime}}{2} \nabla_{T} \psi
$$

Expand it in the basis of the currents $J_{\mu 5}^{a}$ of eq. (2.4):

$$
J_{\mu S}^{l}=\sum_{a} \operatorname{Tr}\left(\tau^{i} t^{a}\right) J_{\mu S}^{a}
$$

In order to obtain the mass matrix for the weak vector bosons, consider the $q \rightarrow 0$ limit of the Fourier transform of the electroweak and axial current-current correlation function,

$$
\mathrm{FT}\left\langle 0\left|\mathrm{~T}\left(J_{\mu 5}^{\imath}(x) J_{\mu 5}^{\prime}(0)\right)\right| 0\right\rangle
$$

Using

$$
\underset{q \rightarrow 0}{\mathrm{FT}}\left\langle 0\left|\mathrm{~T}\left(J_{\mu 5}^{a}(x) J_{\mu 5}^{b}(0)\right)\right| 0\right\rangle=F_{\mathrm{T}}^{2} \delta_{a b}
$$

and the expansion $(2.10)$, we obtain

$$
\underset{q \rightarrow 0}{\mathrm{FT}}\left\langle 0\left|\mathrm{~T}\left(J_{\mu 5}^{\prime}(x) J_{\mu 5}^{\prime}(0)\right)\right| 0\right\rangle=4 F_{\mathrm{T}}^{2} \delta_{l j} .
$$

The factor of 4 is simply the number of electroweak technidoublets. This axial current-current correlation function together with eqs. (2.7) and (2.8) yields the following weak vector boson mass matrix:

$$
M^{2}=\left(\begin{array}{cccc}
W^{+} & W^{-} & W^{3} & B \\
0 & 0 & 0 & 0 \\
0 & 1 & 0 & 0 \\
0 & 0 & 1 & t \\
t^{2}
\end{array}\right){ }_{\frac{1}{4} g_{2}^{2} F_{\mathrm{T}}^{2} N_{\mathrm{TD}},}
$$

where $t=g_{1} / g_{2}=\tan \theta_{\mathrm{W}}$ and $N_{\mathrm{TD}}=4=$ number of technidoublets.

From this we obtain $M_{\mathrm{W}}=\frac{1}{2} g_{2} F_{\mathrm{T}} \sqrt{N_{\mathrm{TD}}}=g_{2} F_{\mathrm{T}}$ and $M_{\mathrm{W}}=M_{\mathrm{Z}} \cos \theta_{\mathrm{W}}$. Using the correct value for the $\mathrm{W}^{ \pm}$and $\mathrm{Z}^{0}$ masses, we obtain

$$
F_{\mathrm{T}} \simeq 125 \mathrm{GeV} \text {. }
$$

Next we want to find the mass of the technirho. This mass sets the scale of the technihadron masses. To find it consider the dimensionless ratio $\mathrm{m}_{\rho} / f_{\pi}$. For QCD this ratio has the value

$$
\frac{m_{\rho}}{f_{\pi}} \simeq \frac{773}{93} \simeq 8.3
$$


However, this number depends on the size $N$ of the color group SU(N) like

$$
\frac{m_{\rho}}{f_{\pi}} \propto \frac{1}{\sqrt{N}},
$$

where $N=3$ for QCD. The reason for this is that $m_{\rho}$ is independent of $N$, whereas $f_{\pi}$ grows as $\sqrt{ } N$ [15]. The latter is easily seen to follow from the QCD version of eq. (2.5). Therefore in order to find $m_{\rho_{\mathrm{T}}}$ we have to scale the $N$-independent ratio

$$
\frac{m_{\rho}}{f_{\pi}} \sqrt{ } 3 \simeq 14.4 \simeq \frac{m_{\rho_{\mathrm{T}}}}{F_{\mathrm{T}}} \sqrt{ } N
$$

Thus,

$$
m_{\rho_{\mathrm{T}}} \simeq \frac{14.4}{\sqrt{N}} F_{\mathrm{T}} \simeq \sqrt{\frac{4}{N}} 900 \mathrm{GeV},
$$

where again $N$ is the size of the technicolor group.

Note that the $N$-dependence of the pseudo-Nambu-Goldstone masses in table 1 arises from the $N$-dependence of $m_{\rho \mathrm{T}}$. Also, since $F_{\mathrm{T}}$ is fixed [eq. (2.15)] it should not be considered to scale with $N$ in the results.

Armed with the previous definitions and scales we can now estimate the masses of the four lightest pseudos. The neutral pseudos (axion, paraxion) do not receive any mass from $S U(3) \times S U(2)_{L} \times U(1)_{Y}$ forces. In order to avoid massless axions they must, however, receive mass from other sources [16]. The simplest possible sources of axionic mass are the standard SU(4) Pati-Salam generators which mediate both quark-lepton and techniquark-technilepton transitions [7]. The charged axions, on the other hand, receive their mass from both Pati-Salam and electroweak forces. The contribution of the electroweak forces to their mass has been calculated in refs. $[4,11,12]$ (see table 1). (See appendix A for the details of this calculation.) Now we shall evaluate the contribution of the Pati-Salam generators to the mass of these pseudos.

Using Dashen's formula [17], we have

$$
\begin{aligned}
M_{a b, p}^{2} & \equiv\left\langle\pi_{\mathrm{T}}^{a}\left|\delta \mathscr{L}^{p}\right| \pi_{\mathrm{T}}^{b}\right\rangle \\
& =\frac{1}{F_{\mathrm{T}}^{2}}\left\langle 0\left|\left[Q_{5}^{a},\left[Q_{5}^{b}, \delta \mathscr{L}^{p}\right]\right]\right| 0\right\rangle,
\end{aligned}
$$

where

$$
\delta \mathscr{L}^{p} \equiv \frac{1}{2} g_{\mathrm{PS}}^{2} \int \frac{\mathrm{d}^{4} q}{(2 \pi)^{4}} \frac{-g^{\mu \nu}}{\left(q^{2}-m_{p}^{2}\right)} \int \mathrm{d}^{4} x \mathrm{e}^{\imath q x} \mathrm{~T}\left(J_{\mu}^{p}(x) J_{\nu}^{p}(0)\right) .
$$

$J_{\mu}^{p}(x)$ is a leptoquark Pati-Salam current, $g_{\mathrm{PS}}$ is the Pati-Salam coupling constant, $m_{p}$ is the leptoquark mass ( $p$ labels the massive leptoquark generators), $Q_{5}^{a}$ is the axial 
charge constructed from the currents of eq. (2.4), and $\delta \mathscr{L}^{p}$ is the second-order Pati-Salam lagrangian. In the limit that $m_{p}$ is much larger than the technihadronic scale $(1 \mathrm{TeV})$ [which is justified in the discussion preceding eq. (2.36)], we can approximate eq. (2.21) by

$$
\delta \mathscr{L}^{p} \equiv \frac{g_{\mathrm{PS}}^{2}}{2 m_{p}^{2}} \mathrm{~T}\left(J_{\mu}^{p}(0) J_{\mu}^{p}(0)\right) .
$$

After applying the double commutator of eq. (2.20), we obtain

$$
\begin{aligned}
M_{a b, p}^{2}= & \frac{4 g_{\mathrm{PS}}^{2}}{F_{\mathrm{T}}^{2} m_{p}^{2}} \sum_{c d}\left\{\operatorname{Tr}\left(t_{c}\left[t_{b},\left[t_{a}, V_{p}\right]\right]\right) \operatorname{Tr}\left(t_{d} V_{p}\right) I_{c d}^{1}\right. \\
& \left.+\operatorname{Tr}\left(t_{c}\left[t_{a}, V_{p}\right]\right) \operatorname{Tr}\left(t_{d}\left[t_{b}, V_{p}\right]\right) I_{c d}^{2}\right\},
\end{aligned}
$$

where $V_{P}$ is defined by

$$
J_{\mu}^{p} \equiv \bar{\psi} \gamma_{\mu} V_{p} \nabla_{T} \psi
$$

and $I^{1,2}$ are given by

$$
\begin{aligned}
I_{c d}^{1} & \equiv\left\langle 0\left|\bar{\psi} \gamma_{\mu} t_{c} \nabla_{\mathrm{T}} \psi \bar{\psi} \gamma^{\mu} t_{d} \nabla_{\mathrm{T}} \psi\right| 0\right\rangle, \\
I_{c d}^{2} & \equiv\left\langle 0\left|\bar{\psi} \gamma_{\mu} \gamma_{5} t_{c} \nabla_{\mathrm{T}} \psi \bar{\psi} \bar{\psi} \gamma^{\mu} \gamma_{5} t_{d} \nabla_{\mathrm{T}} \psi\right| 0\right\rangle .
\end{aligned}
$$

We now assume that the vacuum state dominates the sum over intermediate states in eq. (2.25). This approximation has been substantiated by Shifman et al. [18] in QCD. We find

$$
\begin{aligned}
& I_{c d}^{1} \simeq \frac{1}{2} \delta c d\left(\frac{1}{8}\left\langle\bar{\psi} \nabla_{\mathrm{T}} \psi\right\rangle\right)^{2}, \\
& I_{c d}^{2}=-I_{c d}^{1} .
\end{aligned}
$$

Substituting (2.26) into (2.23) and using the algebraic identities

$$
\begin{gathered}
\operatorname{Tr}\left(t_{c}\left[t_{a}, V_{p}\right]\right)=-i f_{a c d} \operatorname{Tr}\left(t_{d} V_{p}\right), \\
\operatorname{Tr}\left(t_{c}\left[t_{b},\left[t_{a}, V_{p}\right]\right]\right)=-f_{b c d} f_{a d e} \operatorname{Tr}\left(t_{e} V_{p}\right),
\end{gathered}
$$

we obtain

$$
M_{a b, p}^{2}=\frac{g_{\mathrm{PS}}^{2} C_{a b, p}}{F_{\mathrm{T}}^{2} m_{p}^{2}}\left(\frac{1}{8}\left\langle\bar{\psi} \nabla_{T} \psi\right\rangle\right)^{2},
$$

where the Clebsch $C_{a b, p}$ is given by

$$
C_{a b, p} \equiv 4 f_{a c d} f_{b c e} \operatorname{Tr}\left(t_{d} V_{p}\right) \operatorname{Tr}\left(t_{e} V_{p}\right) .
$$

Let us now use the above formalism to evaluate the contribution of the Pati-Salam forces to the light pseudos. Consider first the neutral paraxion $\mathbf{a}_{\mathrm{T}}^{\mathbf{0}}$. In this case

$$
t^{a} \equiv \sqrt{\frac{1}{2}}\left(-\frac{1}{2} \frac{\lambda_{15}}{0}-\frac{0}{\frac{1}{2}} \frac{0}{\lambda_{15}}\right),
$$


where $\frac{1}{2} \lambda a(a=1, \ldots, 15)$ are the $4 \times 4$ Pati-Salam matrices, i.e.

$$
\frac{1}{2} \lambda_{15}=\frac{1}{2 \sqrt{6}}\left(\begin{array}{cccc}
1 & & & \\
& 1 & & \\
& & 1 & \\
& & & -3
\end{array}\right),
$$

and the leptoquark generators $V_{p}$ are

$$
V_{p} \equiv\left(\begin{array}{cc}
\frac{1}{2} \lambda_{p} & 0 \\
0 & \frac{1}{2} \lambda_{p}
\end{array}\right), \quad(p=9, \ldots, 14) .
$$

Using (2.30)-(2.32) we find $\sum_{p} C_{a b, p}=8$ and thus

$$
M_{\mathrm{a}_{\mathrm{T}}}^{2} \equiv \sum_{p=9}^{14} m_{a a, p}^{2}=\frac{8 g_{\mathrm{PS}}^{2}}{F_{\mathrm{T}}^{2} m_{p}^{2}}\left(\frac{1}{8}\left\langle\bar{\psi} \pi_{\mathrm{T}} \psi\right\rangle\right)^{2} .
$$

In order to evaluate the above expression numerically we need to know both (i) $\frac{1}{8}\left\langle\bar{\psi} \nabla_{\mathrm{T}} \psi\right\rangle$ and (ii) $\mathrm{g}_{\mathrm{PS}}^{2} / \mathrm{m}_{\mathrm{p}}^{2}$.

(i) We obtain $\frac{1}{8}\left\langle\bar{\psi} \rrbracket_{T} \bar{\psi}\right\rangle$ by simple scaling arguments. We have

$$
\frac{1}{8}\left\langle\bar{\psi} \nabla_{\mathrm{T}} \psi\right\rangle=N\left(\frac{F_{\mathrm{T}}}{f_{\pi}} \sqrt{\frac{3}{N}}\right)^{3} \frac{1}{3}\left\langle\bar{q} \nabla_{c} q\right\rangle .
$$

Using $\left\langle\bar{q} \mathbb{1}_{c} q\right\rangle \simeq(250 \mathrm{MeV})^{3}$ we find

$$
\frac{1}{8}\left\langle\vec{\psi}_{\mathrm{T}} \psi\right\rangle=\sqrt{\frac{3}{N}}(330 \mathrm{GeV})^{3}
$$

(ii) A lower bound on $m_{p}$ can be estimated as follows. The leptoquark Pati-Salam generators are expected to mediate the highly suppressed decay $K_{L} \rightarrow \mu^{ \pm} e^{\mp}$. Thus, the absence of such a decay sets a lower bound on the mass $m_{p}$ of the leptoquark generator. This bound is estimated in detail in appendix B. Here we only quote the result which is

$$
m_{p} / g_{\mathrm{PS}}>310 \mathrm{TeV} .
$$

We thus obtain the following contribution to the $a_{T}^{0}$ mass

$$
\begin{aligned}
M_{\mathrm{a} \frac{3}{\mathrm{~T}}}^{2} & \simeq \frac{3}{N} \frac{g_{\mathrm{PS}}^{2}}{m_{p}^{2}}\left(6.6 \times 10^{11} \mathrm{GeV}^{4}\right) \\
& \leqslant\left(\sqrt{\frac{4}{\mathrm{~N}}} 2.54 \mathrm{GeV}\right)^{2} .
\end{aligned}
$$

A similar computation for the contribution of Pati-Salam forces to the mass of the axion $M_{\mathrm{a}}^{2}{ }^{3}$ and to the mass of the charged axion $\delta M_{\mathrm{a}_{\mathrm{T}}}^{2_{+}}$yields

$$
\delta M_{\mathrm{a}_{\mathrm{T}}^{+}}^{2}=M_{\mathrm{a}_{\mathrm{T}}^{3}}^{2}=\frac{3}{4} M_{\mathrm{a}_{\mathrm{T}}^{0}}^{2} .
$$


Thus we see that the neutral axions are expected to have a mass which does not exceed $2.5 \mathrm{GeV}$. Moreover, this contribution to the mass of the charged axions is negligible compared to their electroweak mass.

We should emphasize that the lightness of the axions is not necessarily inescapable. For example, if we had assumed that the Pati-Salam generators which cause transitions between techniquarks and technileptons are different than those which cause transitions between quarks and leptons, then the mass of the axions is a priori arbitrary, because we cannot impose the limit 2.36.

\section{Decays of pseudos}

In this section we discuss the dominant decay modes of the pseudos listed in table 1. We shall consider the pseudos in the following order: color octets, triplets, and singlets.

\subsection{COLOR OCTETS}

In table 2 we list some possible decay modes of the color octet pseudos. We shall evaluate the decay rates for the two-body decays which are expected to be dominant.

Let us first consider the decays of the octets into two gauge bosons (fig. 1a). To do this we shall use a more general formalism which applies to the decay of any pseudo into two gauge bosons. Let $\Pi_{\mathrm{T}}^{a}$ be the pseudo defined by eq. (2.5). Let $J_{\mu}=V_{\mu}+A_{\mu}$ be any $\mathrm{SU}(3)_{\mathrm{C}} \times \mathrm{SU}(2)_{\mathrm{L}} \times \mathrm{U}(1)_{Y}$ gauge current where

$$
V_{\mu}=\bar{\psi} \gamma_{\mu} \Gamma_{\mathrm{V}} \mathbb{T}_{\mathrm{T}} \psi
$$

and

$$
A_{\mu}=\bar{\psi} \gamma_{\mu} \gamma_{5} \Gamma_{\mathrm{A}} \eta_{\mathrm{T}} \psi
$$

and $\Gamma_{\mathrm{V}}, \Gamma_{\mathrm{A}}$ are matrices in the $\mathrm{SU}(8)$ space defined by eq. (2.3). (Note that the matrices $\Gamma_{\mathrm{V}}$ and $\Gamma_{\mathrm{A}}$ also include the gauge coupling constant.) Consider now the decay of $\Pi_{\mathrm{T}}^{a}$ into two gauge bosons that couple to $J_{\mu}^{1}$ and $J_{\mu}^{2}$. The amplitude $T$ for this process is given by

$$
T=\lim _{q \rightarrow 0} \frac{1}{F_{\mathrm{T}}} \int \mathrm{d}^{4} x \mathrm{~d}^{4} y \mathrm{e}^{\imath q x} \partial^{\mu}\left\langle 0\left|\mathrm{~T}\left(J_{\mu 5}^{a}(x) J_{\alpha}^{1}(y) J_{\beta}^{2}(0)\right)\right| 0\right\rangle \varepsilon_{1}^{* \alpha}\left(p_{1}\right) \varepsilon_{2}^{* \beta}\left(p_{2}\right) \mathrm{e}^{-\imath p_{1} y} .
$$

The Green function $\left\langle 0\left|T\left(J_{\mu 5}^{a}(x) J_{\alpha}^{1}(y) J_{\beta}^{2}(0)\right)\right| C\right\rangle$ to lowest order in electroweak interactions is given by the Adler-Bell-Jackiw anomaly [19]. Notice that only the VVA and AAA terms contribute. Following ref. [19] we obtain

$$
T=\frac{S}{2 \pi^{2} F_{\mathrm{T}}} \varepsilon_{\mu \nu \rho \sigma} p^{1 \mu} p^{2 \sigma} \varepsilon_{1}^{* \nu} \varepsilon_{2}^{* o},
$$


TABLE 2

Dominant decay modes of color octet pseudos which conserve C-conjugation, parity and the $\mathrm{SU}(2)_{V}$ techni-1sospin symmetry

\begin{tabular}{clc}
\hline Pseudo & Decay modes & Rates $(\mathrm{MeV})$ \\
\hline$\eta_{\mathrm{T}}^{a}$ & $\overline{\mathrm{t}}+\mathrm{t}$ & $1100 \sqrt{\frac{4}{N}}$ \\
& $\mathrm{G}+\mathrm{G}$ & $60 \sqrt{\frac{1}{4} N}$ \\
& $\mathrm{G}+\gamma$ & $06 \sqrt{\frac{1}{4} N}$ \\
& $\mathrm{G}+\mathrm{Z}_{0}$ & $2 \sqrt{\frac{1}{4} N}$ \\
& $\mathrm{G}+\mathrm{G}+\mathrm{G}$ & \\
& $\mathrm{G}+\mathrm{G}^{\mathrm{T}} \mathrm{a}_{\mathrm{T}}^{0}$ & \\
& $\mathrm{G}+\mathrm{Z}^{0}+\mathrm{Z}^{0}$ & \\
& $\mathrm{G}+\mathrm{W}^{+}+\mathrm{W}^{-}$ & $5.4 \sqrt{\frac{1}{4} N}$ \\
& $\mathrm{G}+\mathrm{Z}^{0}+\gamma$ & $2.4 \sqrt{\frac{1}{4} N}$ \\
& $\mathrm{G}+\gamma$ & \\
\hline$\pi_{\mathrm{T}}^{\mathrm{a}^{3}}$ & $\mathrm{G}+\mathrm{Z}_{0}$ & \\
& $\mathrm{G}+\mathrm{a}_{\mathrm{T}}^{3(0)}+\gamma$ & $1100 \sqrt{\frac{4}{N}}$ \\
& $\mathrm{G}+\mathrm{a}_{\mathrm{T}}^{3(0)}+\mathrm{Z}^{0}$ & \\
& $\mathrm{G}+\mathrm{a}_{\mathrm{T}}^{-}+\mathrm{W}^{+}$ & $48 \sqrt{\frac{1}{4} N}$ \\
& $\overline{\mathrm{t}}+\mathrm{t}$ & $375 \sqrt{\frac{4}{N}}$ \\
\hline$\pi_{\mathrm{T}}^{\mathrm{a}^{+}}$ & $\mathrm{G}+\mathrm{W}^{+}$ & \\
& $\mathrm{t}+\overrightarrow{\mathrm{b}}$ & \\
\hline
\end{tabular}

$\mathrm{G}$ stands for gluon, $\mathrm{t}$ and $\mathrm{b}$ are the top and bottom quarks respectively.

where $S$ is given by

$$
S=\frac{1}{2} N \operatorname{Tr} t^{a}\left(\left\{\Gamma_{\mathrm{V}}^{1}, \Gamma_{\mathrm{V}}^{2}\right\}+\left\{\Gamma_{\mathrm{A}}^{1}, \Gamma_{\mathrm{A}}^{2}\right\}\right),
$$

and $N=\operatorname{Tr} \nabla_{\mathrm{T}}$. The rate is then given by

$$
\Gamma=S^{2} \frac{1}{256 \pi^{5}} \frac{m^{3}}{F_{\mathrm{T}}^{2}},
$$

where $m$ is the mass of the pseudo ${ }^{\star \star \star}$.

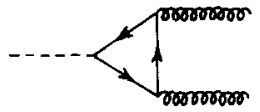

(a)

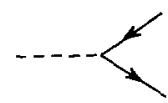

(b)

Fig. 1 Relevant graphs for the two-body decays, and (backwards) the production, of the color octet and singlet pseudos

* Eq. (3 5) is correct for decays into two identical gauge bosons. For decays into non-ıdentical particles we must multiply by a factor of 2 .

$\star$ Throughout this paper we always approximate all phase spaces contributions by those of massless particles. 
We can check this formalism for the classic case of $\pi^{0} \rightarrow \gamma \gamma$ where in this case

$$
\begin{gathered}
\psi=\left(\begin{array}{l}
\mathrm{u} \\
\mathrm{d}
\end{array}\right) ; \quad t^{3} \otimes \mathbb{1}_{\text {color }}=\frac{1}{2} \tau^{3} \otimes \mathbb{1}_{\text {color }}=\left(\begin{array}{cc}
\frac{1}{2} & 0 \\
0 & -\frac{1}{2}
\end{array}\right)_{\text {flavor }} \otimes \mathbb{1}_{\text {color }}, \\
\Gamma_{\mathrm{V}}^{1}=\Gamma_{\mathrm{V}}^{2}=\left(\begin{array}{cc}
\frac{2}{3} e & 0 \\
0 & -\frac{1}{3} e
\end{array}\right)_{\text {flavor }} \otimes v_{\text {color }}, \quad \Gamma_{\mathrm{A}}^{1}=\Gamma_{\mathrm{A}}^{2}=0 .
\end{gathered}
$$

Then $S$ is equal to $\frac{1}{2} e^{2}$ and the decay rate is

$$
\Gamma_{\pi^{0} \rightarrow \gamma \gamma}=\frac{\alpha^{2}}{64 \pi^{3}} \frac{m_{\pi}^{3}}{f_{\pi}^{2}}=7.47 \mathrm{eV} .
$$

Let us now calculate the total decay rate for the process $\eta_{\mathrm{T}}^{a} \rightarrow \mathrm{G}^{b}+\mathrm{G}^{c}$. In this case

$$
\begin{aligned}
& t^{a}=\sqrt{\frac{1}{2}}\left(\begin{array}{ccc}
\frac{1}{2} \lambda_{a} & \\
& 0 & \\
& \frac{1}{2} \lambda_{a} \\
& & 0
\end{array}\right), \\
& \Gamma_{\mathrm{V}}^{b}=\sqrt{2} g_{3} t^{b}, \quad \Gamma_{\mathrm{A}}^{b}=0,
\end{aligned}
$$

where $a, b, c=1,2, \ldots, 8 ; \lambda^{a}$ are the Gell-Mann SU(3) color matrices and $g_{3}$ is the SU(3) coupling constant. Thus,

$$
\begin{gathered}
S_{a b c}=\frac{N g_{3}^{2}}{2 \sqrt{2}} d_{a b c}, \\
\Gamma_{\eta_{\mathrm{T}}^{a} \rightarrow \mathrm{GG}}=\sum_{b, c} \Gamma_{\eta_{\mathrm{T}}^{a} \rightarrow \mathrm{G}^{b} \mathrm{G}^{c}} \simeq \frac{5 N^{2}}{384 \pi^{3}} \alpha_{3}^{2} \frac{m_{\eta_{\mathrm{T}}^{a}}^{3}}{F_{\mathrm{T}}^{2}} \simeq 60 \mathrm{MeV} \sqrt{\frac{1}{4} N} .
\end{gathered}
$$

Note that here the loop is made of technifermions, and the number of loops contributing to the amplitude is ( 2 techniflavors $) \times(3$ colors $) \times(N$ technicolors $)$. We took $\alpha_{3} \simeq 10^{-1}$ and $m_{m_{\mathrm{T}}} \simeq 240 \sqrt{4 / \mathrm{N}} \mathrm{GeV}$.

In table 2 we have listed the analogous results for the two gauge boson decays of all the color octets. Let us now consider the decay of the color octet pseudos into a pair of fermions (fig. 1b) which is expected to be the dominant decay mode. To estimate this process we have assumed that the effective Yukawa coupling $g_{y}$ of the octets to a pair of fermions $f$ and $f^{\prime}$ is given by (using either a Goldberger-Treiman type argument, or from models which give quark masses and couplings)

$$
g_{\mathrm{y}} \sim \frac{m_{\mathrm{f}}+m_{\mathrm{f}^{\prime}}}{F_{\mathrm{T}}}
$$

In order to evaluate this process more accuratedy, however, one needs more model-dependent information on the extended technicolor sector of this theory. In the absence of such information we believe, nevertheless, that the assumption (3.11) 


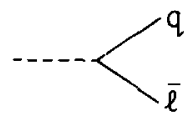

Fig. 2. Decay (production) of the leptoquark bosons.

for the value of $g_{y}$ is a valid order of magnitude estimate. With this caveat, we find the rate to be

$$
\Gamma_{\text {pseudo } \rightarrow \text { ff' }}=\frac{g_{\mathrm{y}}^{2} m_{\text {pseudo }}}{16 \pi}
$$

The results are listed in table 2 .

\section{COLOR TRIPLETS}

The dominant decay mode of the color triplet pseudos is into a quark and an antilepton (fig. 2). The computation is identical to that of subsect. 3.1. The results are listed in table 3 .

\section{COLOR SINGLETS}

There are 4 color singlet pseudos in this scenario $\left[\mathrm{a}_{\mathrm{T}}^{0}, \mathrm{a}_{\mathrm{T}}^{3}, \mathrm{a}_{\mathrm{T}}^{ \pm}\right]$. They are especially interesting since they are expected to be light (see table 4). An important difference between this scenario and the standard Higgs scenario is that all of the light axions are pseudoscalars. In fact, in these theories the standard Higgs particle is replaced by

TABLE 3

Decays of color triplet pseudos

\begin{tabular}{lcc}
\hline Pseudo & Decay modes & Rates $(\mathrm{MeV})$ \\
\hline $\bar{E} U$ & $\mathrm{t}+\tau^{+}$ & $190 \sqrt{\frac{4}{N}}$ \\
\hline$\sqrt{\frac{\mathrm{T}}{2}(\bar{E} D-\bar{N} U)}$ & $\mathrm{t}+\bar{\nu}$ & $95 \sqrt{\frac{4}{N}}$ \\
& $\mathrm{~b}+\tau^{+}$ & $4 \sqrt{\frac{4}{N}}$ \\
\hline $\bar{N} D$ & $\mathrm{~b}+\bar{\nu}$ & $8 \sqrt{\frac{4}{N}}$ \\
\hline$\sqrt{\frac{\mathrm{T}}{2}(\bar{E} D+\bar{N} U)}$ & $\mathrm{t}+\bar{\nu}$ & $95 \sqrt{\frac{4}{N}}$ \\
& $\mathrm{~b}+\tau^{+}$ & $4 \sqrt{\frac{4}{N}}$ \\
\hline
\end{tabular}


TABLE 4

Contributions to the masses squared of the color singlet pseudos

\begin{tabular}{cccc}
\hline & \multicolumn{2}{c}{$M^{2}\left(\mathrm{GeV}^{2}\right)$} & Total \\
\hline Pseudo & Electroweak & Pat1-Salam & $m \leqslant 2.54 \mathrm{GeV}$ \\
$\mathbf{a}_{\mathrm{T}}^{\mathbf{3}}$ & 0 & $(2.54)^{2}$ & $m \leqslant 216 \mathrm{GeV}$ \\
$\mathrm{a}_{\mathrm{T}}^{ \pm}$ & 0 & $(2.16)^{2}$ & $m \leq 8 \mathrm{GeV}$ \\
& $(7.7)^{2}$ & $(2.16)^{2}$ & $m=8$ \\
\hline
\end{tabular}

a composite scalar with a mass of order $1 \mathrm{TeV}$. Thus the discovery of a light scalar would be strong evidence against theories of dynamical symmetry breaking.

In table 5 we list some possible decay modes of the color singlet pseudos. The calculation of the decay rates into two body modes is identical to those discussed in subsect. 3.1. For example, for the total decay rate for $a_{T}^{0} \rightarrow G^{a}+G^{b}$ we find explicitly

$$
\sum_{a b} \Gamma_{z_{\mathrm{T}} \rightarrow \mathrm{G}^{a} \mathrm{G}^{b}}=\left\{\frac{\alpha_{3}^{2}}{6 \pi^{3} F_{\mathrm{T}}^{2}}\right\} m_{a}^{3}\left(\frac{1}{4} N\right)^{2} .
$$

The results are given in table 5. The following comments are in order: this model has the same number of color singlet spinless bosons as the non-minimal two-Higgs,

\section{TABLE 5}

Domınant decays of light color singlet pseudos

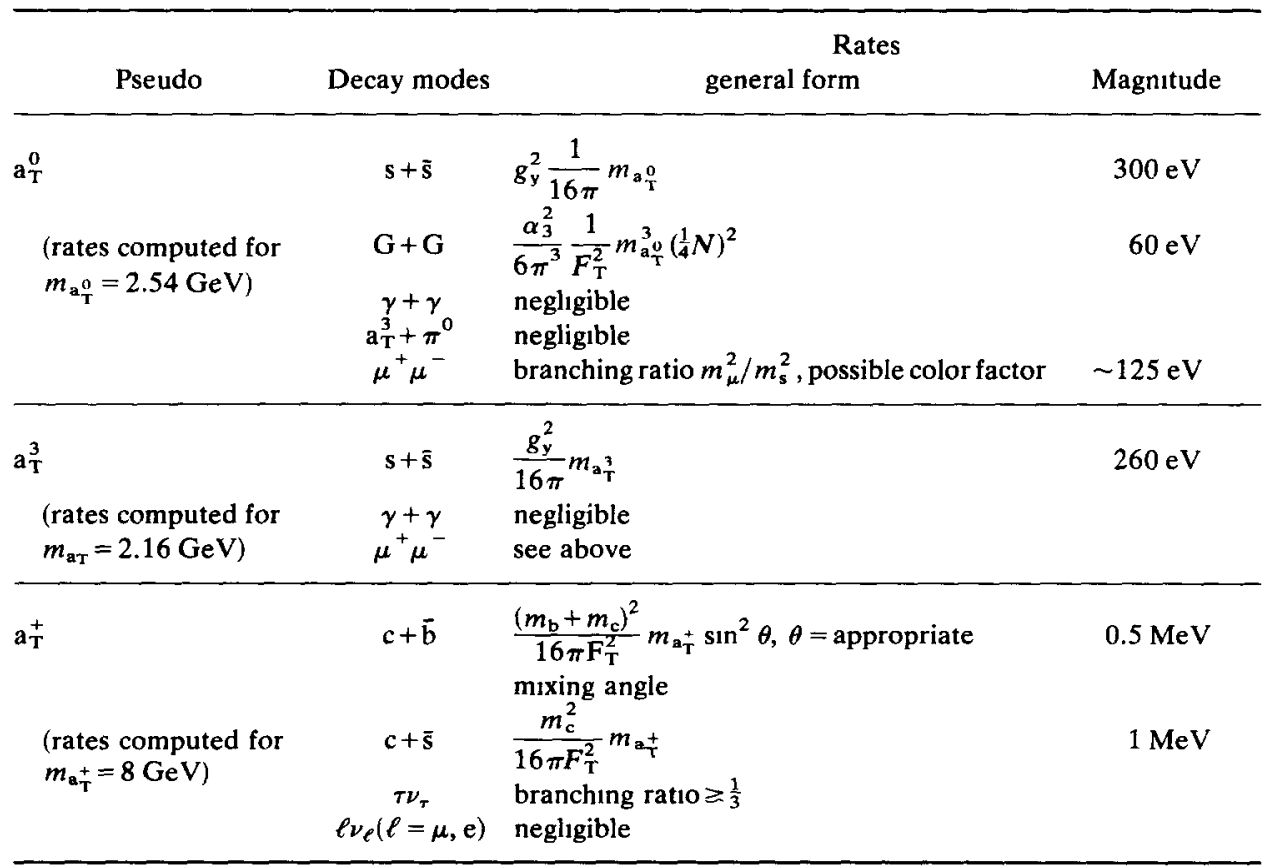


Glashow-Weinberg-Salam model [1]. The important difference is that the twoHiggs model has two neutral scalars and one neutral pseudoscalar whose tree-level Yukawa couplings are parity conserving. In contrast, in a dynamical scenario some of the Yukawa couplings of the light neutral axions are expected to violate parity. This is in fact a mechanism for splitting up and down quark masses in extended technicolor scenarios $[20,7]$. Thus one would expect to see some parity-violating fermionic decays of light, neutral axions. In contrast to the fermionic decays, the two-photon and two- and three-gluon decay modes of the neutral axion and paraxion are to lowest order parity conserving. These decays can thus be used to determine the parity of the neutral axions $[10,14]$. For example, since they are pseudoscalars they cannot decay into four gluons.

\section{Production cross sections}

In this section we consider some processes which give rise to single production of technihadrons.

We discuss the following processes:

(a) $\bar{p} \bar{p}(\mathrm{pp}) \rightarrow \eta_{\mathrm{T}}^{a}+\mathrm{X}$

(b) $\mathrm{p} \overline{\mathrm{p}}(\mathrm{pp}) \rightarrow \mathrm{a}_{\mathrm{T}}^{0}+\mathrm{X}$,

(c) $\mathbf{p} \overline{\mathrm{p}} \rightarrow \rho_{\mathrm{T}}^{a}+\mathrm{X}$.

These processes may lead to the production of technihadrons in the colliding Tevatron machines.

41. $\mathrm{p} \overline{\mathrm{p}}(\mathrm{pp}) \rightarrow \eta_{\mathrm{T}}^{a}+\mathrm{X}$

The cross section $\mathrm{d} \sigma / \mathrm{d} y$ for this process (see fig. 3 ) is given by [21]

$$
\frac{\mathrm{d} \sigma}{\mathrm{d} y}=\pi^{2} \frac{\Gamma\left(\eta_{\mathrm{T}}^{a} \rightarrow \mathrm{GG}\right)}{m_{\eta_{\mathrm{T}}}^{3}} G\left(x_{1}\right) G\left(x_{2}\right),
$$

where $G(x)$ is the gluon distribution function in the proton and the kinematic variables (fig. 3) are defined in the standard fashion, i.e. $x_{1}$ and $x_{2}$ are the fraction of

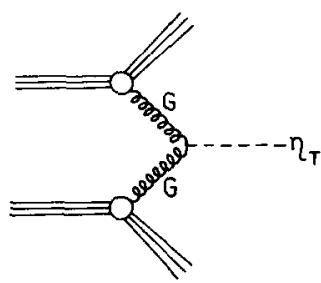

Fig 3 Production mechanism for $\eta_{\mathrm{T}}^{a}$.

\footnotetext{
* In order to obtain the decay rate into any specific channel using our production cross sections, one simply multiplies our result by the branching ratio for the particular channel of interest.
} 
longitudinal momentum carried by the gluons and $y$ is the rapidity of $\eta_{\mathrm{T}}$. They are related by (see fig. 3 ).

$$
\begin{gathered}
y \equiv \frac{1}{2} \ln \frac{E_{\eta_{\mathrm{T}}}+P_{\eta_{\mathrm{T}}}^{\mathrm{L}}}{E_{\eta_{\mathrm{T}}}-P_{\eta_{\mathrm{T}}}^{\mathrm{L}}} \simeq \frac{1}{2} \ln \frac{x_{1}}{x_{2}}, \\
x_{1}=\frac{m_{\eta_{\mathrm{T}}}}{\sqrt{s}} \mathrm{e}^{y}, \quad x_{2}=\frac{m_{\eta_{\mathrm{T}}}}{\sqrt{s}} \mathrm{e}^{-y},
\end{gathered}
$$

where $\sqrt{ } s$ is the total c.m. energy and

$$
\int_{0}^{1} \mathrm{~d} x G(x) \simeq \frac{1}{2} \text {. }
$$

Although $G(x)$ is not known we shall use the form

$$
G(x)=3(1-x)^{5} \text {. }
$$

Using eqs. (4.1) and (3.10) we find

$$
\begin{aligned}
\left.\frac{\mathrm{d} \sigma}{\mathrm{d} y}\right|_{y=0} & =\frac{15}{128 \pi} \frac{\alpha_{3}^{2}}{F_{\mathrm{T}}^{2}}\left(1-\frac{m_{\eta_{\mathrm{T}}}}{\sqrt{s}}\right)^{10} N^{2} \\
& \approx 160 \times 10^{-36} \mathrm{~cm}^{2}\left(1-\frac{m_{\eta_{\mathrm{T}}}}{\sqrt{s}}\right)^{10}\left(\frac{1}{4} N\right)^{2}
\end{aligned}
$$

For $N=4$ and $m_{\eta_{\mathrm{T}}}=240 \mathrm{GeV}$ we obtain the following values for $\mathrm{d} \sigma /\left.\mathrm{d} y\right|_{y=0}$ :

$$
\begin{aligned}
& \sqrt{s}=500 \mathrm{GeV}:\left.\frac{\mathrm{d} \sigma}{\mathrm{d} y}\right|_{y=0}=2 \times 10^{-37} \mathrm{~cm}^{2}, \\
& \sqrt{s}=800 \mathrm{GeV}:\left.\frac{\mathrm{d} \sigma}{\mathrm{d} y}\right|_{y=0}=4.5 \times 10^{-36} \mathrm{~cm}^{2}, \\
& \sqrt{ } s=2000 \mathrm{GeV}:\left.\frac{\mathrm{d} \sigma}{\mathrm{d} y}\right|_{y=0}=44 \times 10^{-36} \mathrm{~cm}^{2} .
\end{aligned}
$$

\section{2. $\mathrm{p} \overline{\mathrm{p}}(\mathrm{pp}) \rightarrow \mathrm{a}_{\mathrm{T}}^{0}+\mathrm{X}$}

This single paraxion production cross section is given by

$$
\left.\frac{\mathrm{d} \sigma}{\mathrm{d} y}\right|_{y=0}=\frac{3 \alpha_{3}^{2}}{16 \pi} \frac{1}{F_{\mathrm{T}}^{2}}\left(1-\frac{m_{\mathrm{a}_{\mathrm{T}}^{0}}}{\sqrt{s}}\right)^{10}\left(\frac{1}{4} N\right)^{2} \simeq 15 \times 10^{-36} \mathrm{~cm}^{2}\left(1-\frac{m_{\mathrm{a}} \frac{0}{\mathrm{~T}}}{\sqrt{s}}\right)^{10}\left(\frac{1}{4} N\right)^{2} .
$$

43. $\mathrm{p} \overline{\mathrm{p}} \rightarrow \rho_{\mathrm{T}}^{a}+\mathrm{X}$

The cross section for this process is given by

$$
\left.\frac{\mathrm{d} \sigma}{\mathrm{d} y}\right|_{y=0}=\frac{16 \pi^{2}}{m_{\rho_{\mathrm{T}}^{\mathrm{a}}}^{3}}\left\{\frac{2}{3} \Gamma_{\rho_{\mathrm{T}}^{\mathrm{a}} \rightarrow \overline{\mathrm{u}} \mathrm{u}} u(x)^{2}+\frac{2}{3} \Gamma_{\rho_{\mathrm{T}}^{\mathrm{a}} \rightarrow \overline{\mathrm{d} d}} d(x)^{2}+\frac{3}{16} \Gamma_{\rho_{\mathrm{T}}^{a} \rightarrow \mathrm{GG}} G(x)^{2}\right\},
$$


where $\Gamma_{\rho_{T \rightarrow \bar{u}}}$ is the total decay rate for $\rho_{\mathrm{T}}^{a}$ into a uu pair, $u(x)$ is the up quark distribution function in a proton and $x=m_{\rho_{T}} / \sqrt{ } s$. In order to evaluate this cross section we must compute the decay rates $\Gamma_{\rho_{T}^{a} \rightarrow \overline{\mathrm{u}} u}, \Gamma_{\rho_{\mathrm{T}}^{a} \rightarrow \overline{\mathrm{dd}}}$ and $\Gamma_{\rho_{\mathrm{T}}^{a} \rightarrow \mathrm{GG}}$. This calculation is done in appendix $\mathrm{C}$. We find

$$
\begin{aligned}
& \Gamma_{\rho_{\mathrm{T}}^{\mathrm{a}} \rightarrow \overline{\mathrm{u}} \mathrm{u}}=\Gamma_{\rho_{\mathrm{T}}^{\mathrm{a}} \rightarrow \overline{\mathrm{d} d}}=\frac{4 \pi}{3} \alpha_{3}^{2} \frac{F_{\rho_{\mathrm{T}}}^{2}}{m_{\rho_{\mathrm{T}}}}, \\
& \Gamma_{\rho_{\mathrm{T} \rightarrow \mathrm{GG}}^{a}}=2 \pi \alpha_{3}^{2} \frac{F_{\rho_{\mathrm{T}}}^{2}}{m_{\rho_{\mathrm{T}}}} .
\end{aligned}
$$

Using the result of Weinberg's sum rules discussed previously in eq. (A.16) we can relate $F_{\rho}$ to $F_{\mathrm{T}}$. We find

where

$$
F_{\rho}^{2} \equiv \frac{1}{1-\varepsilon} F_{\mathrm{T}}^{2}
$$

$$
\varepsilon \equiv \frac{m_{\rho_{\mathrm{T}}}^{2}}{m_{\mathrm{A}_{\mathrm{T}}}^{2}} \simeq \frac{1}{2} .
$$

Finally combining the results of eqs. (4.8) and (4.9) we find the differential cross section for $\sqrt{ } s=2000 \mathrm{GeV}$, using $u^{2}(x)+d^{2}(x) \simeq 0.06$ :

$$
\left.\frac{\mathrm{d} \sigma}{\mathrm{d} y}\right|_{y=0} \approx 6 \times 10^{-36} \mathrm{~cm}^{2} \text {. }
$$

Note this scales as $u^{2}\left(m_{\rho_{\mathrm{T}}} / \sqrt{ } s\right) / m_{\rho \mathrm{T}}^{4}$ so it would get considerably larger if $m_{\rho_{\mathrm{T}}}$ decreased a little.

\section{Implications for experiment}

Here we use the numbers computed in the previous sections to emphasize the consequences for experiment.

\subsection{CHARGED AXIONS}

We expect $m_{\mathrm{a}} \simeq 8 \mathrm{GeV}$.

If the basic ideas of technicolor physics are correct it is likely that charged dynamical scalar bosons (pointlike until a TeV mass scale) exist ${ }^{\star}$ with about this mass. They should be detectable at PETRA/PEP'

This number is made up of $7.7 \mathrm{GeV}$ from electroweak contributions, which should be reliably calculated, and up to $2.2 \mathrm{GeV}$ from Pati-Salam flavor-changing interactions, adding in $m_{\mathrm{a}}^{2}$. The absence of such charged dynamical spin-zero bosons

* One interesting consequence of this is that the decay $t \rightarrow b a_{T}^{+}$will dominate $t$ decay since it is semiweak. Then $\mathrm{a}_{\mathrm{T}}^{+}$mainly gives $\overline{\mathrm{b}} \mathrm{c}, \overline{\mathrm{s}} \mathrm{c}$ and $\tau^{+} \nu_{n}$, so there is a violation of $\tau / \mu / \mathrm{e}$ universality (hard to see since $\tau^{+}$ gives equal $\mu^{+}, \mathrm{e}^{+}$) and the $\mathrm{t}$ decay pattern is not as expected 
would be a strong constraint on constructing theories of dynamical symmetry breaking. So far no model has been constructed without them, but since there is no realistic model yet it is not clear how to interpret this. The situation is somewhat similar to that with nucleon decay and grand unification. There, the simplest ideas suggest the nucleon should decay, and in the simpler theories one can calculate the lifetime. Similarly, here, the simplest scenarios of dynamical symmetry breaking require charged axions with mass about $8 \mathrm{GeV}$. On the other hand, it is possible to construet grand unification schemes with a stable nucleon, and it is possible that much lighter Pati-Salam bosons could exist, increasing the mass of $\mathrm{a}_{\mathrm{T}}^{ \pm}$. But it is most appropriate to be hopeful that $\mathrm{a}_{\mathrm{T}}^{ \pm}$will exist as predicted, and to take the steps to find them.

To detect them the following properties (see also refs. [10,14, 22]) are relevant for $\mathrm{e}^{+} \mathrm{e}^{-} \rightarrow \mathrm{a}_{\mathrm{T}}^{+} \mathrm{a}_{\mathrm{T}}^{-}$.

(a) Scalar bosons have $\frac{1}{4}$ unit of $R, \sin ^{2} \theta$ production angular distribution, and a $\beta^{3}$ threshold behavior.

(b) In this mass range the largest decay modes of $a_{T}^{ \pm}$will be $a_{T}^{+} \rightarrow c \bar{b}, c \bar{s}, \tau \nu_{\tau}$. Whether $c \bar{b}$ or $c \bar{s}$ dominates depends on the quark mixing angles. The ratio will be

$$
\frac{\Gamma(\mathrm{c} \overline{\mathrm{b}})}{\Gamma(\mathrm{c} \overline{\mathrm{s}})} \simeq \frac{m_{\mathrm{b}}^{2}}{m_{\mathrm{c}}^{2}} \sin ^{2} \theta,
$$

where $\theta$ is an appropriate quark mixing angle $\left(\theta=\theta_{2}\right.$ in the most usual parameterization). For $m_{\mathrm{b}}^{2} / m_{\mathrm{c}}^{2} \approx 10, \mathrm{c} \overline{\mathrm{s}}$ is probably the larger mode for typical $\theta(\theta \leqslant 0.05)$, and could dominate for small $\sin ^{2} \theta$. Then $\mathrm{a}_{\mathrm{T}}$ will look like a charmed $\mathrm{F}^{ \pm}$ at a higher mass, with all the modes expected for $\mathrm{F}^{ \pm}$decay. The $\tau \nu_{\tau}$ mode (or any violation of $\tau / \mu /$ e universality) could be a helpful signal - any unusual source of $\tau$ 's should be looked for, and this mode may be the dominant one.

(c) In both hadronic modes one gets 2 strange quarks from each of $\mathrm{a}_{\mathrm{T}}^{+}, \mathrm{a}_{\mathrm{T}}^{-}$, so four (strange quarks) altogether, which will produce four strange particles and/or $\phi, \eta$.

(d) Specific exclusive modes such as $\mathrm{a}_{\mathrm{T}}^{ \pm} \rightarrow \psi \mathrm{F}^{ \pm}, \psi \mathrm{K} \overline{\mathrm{K}}, \psi \mathrm{D} \overline{\mathrm{K}}, \phi \pi^{ \pm}, \mathrm{DK}, \mathrm{DK} \pi, \mathrm{F}^{ \pm} \eta$, $\mathrm{F}^{ \pm} \phi, \mathrm{D} \overline{\mathrm{B}}$, etc. (with $\mathrm{D} \rightarrow \mathrm{D}^{*}$ and other generalizations as well) could be very clear signals, with little background. They also allow spin determination. The last five listed modes are expected to be the dominant ones.

(e) Situations where $\mathrm{a}_{\mathrm{T}}^{+}$(say) decays hadronically, giving a core of hadrons with half the beam energy, and $\mathrm{a}_{\mathrm{T}}^{-}$decays into a semileptonic or leptonic or low multiplicity mode, could perhaps be exploited as a signature. There is room for clever analysis here.

\subsection{PRODUCTION OF COLORED TECHNIETA}

As seen from sect. 4, a lower limit on the cross section for producing the colored technieta state can be reliably calculated through the anomaly contribution. The 
resulting cross sections are big enough to be observable at the next hadron colliders, with about 440 events at colliding Tevatrons (assuming $\mathscr{L}=10^{30} / \mathrm{cm}^{2} \mathrm{sec}$ ) in $10^{7} \mathrm{sec}$, and about 3000 events at Isabelle (assuming $\mathscr{L}=10^{32} / \mathrm{cm}^{2} \mathrm{sec}$ ) in $10^{7} \mathrm{sec}$.

It will dominantly decay to $t \bar{t}$, where $t$ is the heaviest quark, assuming there is one heavier than the $b$; if the $b$ is the heaviest quark the $b \bar{b}$ and $G G$ rates are about equal. To find $\eta_{\mathrm{T}}^{a}$ it will be necessary to do effective mass work with jets. For the GG modes there are two-jet events which must be separated from large background. For $t \bar{t}$ there will be four - and six-jet events, since $t$ will give 2 or 3 jets depending on its mass, with a background of order the signal ${ }^{\star}$. In both cases it will be necessary to reconstruct the mass of $\eta_{\mathrm{T}}^{a}$ (about $240 \mathrm{GeV}$ ) to show convincingly that such a state is found. Since the total widths of $\eta_{T}^{a}$ is $\leqslant 1 \mathrm{GeV}$, the achievable experimental resolution is an important aspect of determining what can be detected. If the theory is qualitatively correct, such a state should appear, with essentially the properties described here.

It is useful to compare the situation with $\eta_{\mathrm{T}}^{a}$ and a standard fundamental Higgs $\mathrm{h}^{\circ}$ (say of the same mass). The production cross section for $\eta_{\mathrm{T}}^{a}$ is larger by a factor,

$$
\sigma\left(\eta_{\mathrm{T}}^{a}\right) / \sigma\left(\mathrm{h}^{0}\right) \simeq 8 N^{2}
$$

$N$ is the dimension of the technicolor group. In summing over fermion loops in the anomaly contribution one has number of loops $=$ (number of techniflavors) $\times$ (number of colors) $\times$ (number of technicolors) for $\eta_{\mathrm{T}}^{a}$, versus (number of heavy quark flavors) $\times$ (number of colors) for $\mathrm{h}^{0}$, and appropriate factors of 2 from the relation between $\langle\phi\rangle$ and $F_{\mathrm{T}}$. The anomaly contribution for $\eta_{\mathrm{T}}^{a}$ is (approximately) larger by a factor $N$ in amplitude, $N^{2}$ in rate. Further, the production cross section is larger by a factor 8 since $\eta_{\mathrm{T}}^{a}$ is in a color octet and it is sufficient to produce any of the 8 states. Thus (if, say, $N=4$ ) it is $8 \times 16=128$ times easier to produce $\eta_{\mathrm{T}}^{a}$ (and test technicolor) than to produce $\mathrm{h}^{0}$ ! For decays, the GG mode is $N^{2}$ larger for $\eta_{\mathrm{T}}^{a}$, so it is expected to be negligible for $h^{0}$ compared to $q \bar{q}$, while for $\eta_{\mathrm{T}}^{a}$ the GG mode is at least $5 \%$, and perhaps large if $t \bar{t}$ is absent.

We have ignored scaling violations in these calculations, which is a reasonable approximation. They have little effect since the important $x$ values are in the range $0.1-0.25$ where the scaling violations are minimal, even for $Q^{2} \simeq m^{2}\left(\eta_{\mathrm{T}}^{a}\right)$. The scaling violations could even enhance the results slightly at colliding Tevatrons, where one might be on the rising part of the curve, while at $400 \mathrm{GeV}$ hadron colliders there will be perhaps a factor of 2 suppression.

\subsection{RECOGNIZING TECHNICOLOR}

There are a number of other ways that the heavy technicolor singlets of table 1 could be produced. We have given an estimate for production of the technirho in

* G L K. thanks S. Wolfram for a helpful conversation about this point 
sect. 4. That estimate, and others one could make, are very model dependent and not necessarily reliable, in contrast to the lower limit on production of technieta from the anomaly contribution. Note, for example, that the technirho rate goes as $1 / m_{\mathrm{T}_{\rho}}^{4}$ so it increases significantly if $m_{\mathrm{T}_{o}}$ is overestimated. With events such as

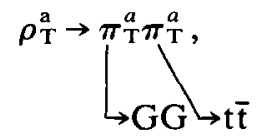

one would see dramatic multijet structure with little QCD background. With the branching ratios and masses of tables $1-5$, and the extensive discussion of refs. $[8,9]$ possible scenarios for hadron collider experiments can be discussed and any interesting events can be interpreted.

\section{4. HIGGS VERSUS TECHNICOLOR}

Distinguishing whether spontaneous symmetry breaking occurs with fundamental scalar bosons (Higgs) or dynamically will not be straight-forward, even after candidate states are found. The light axion states, charged and neutral, will show no structure until probed on a $\mathrm{TeV}$ scale. The differences expected are significant, however, in the mass spectrum, parity structure, and decays, so a detailed study of these properties should suffice. We have remarked on some features in sects 3,4 . Some discussion is given in refs. $[10,14]$. The presence of a light, neutral scalar would probably confirm the Higgs alternative, while finding the charged states around $8 \mathrm{GeV}$ and no neutral scalar would favor technicolor strongly (but charged scalars do occur in both forms of the theory). Finding the technieta with a cross section of at least that of sect. 4 (over 100 times that for a fundamental Higgs) would strongly favor technicolor.

The work of S.D. and of S.R. was supported in part by the National Science Foundation, and that of G.L.K. by the US Department of Energy. G.L.K. appreciates helpful conversations with J. Owens, S. Wolfram, and M. Peskin, and all of us are grateful for stimulating discussions and significant contributions from J. Bjorken. S.D. and S.R. appreciate interesting conversations with L. Susskind and E. Fahri. We thank M. Peskin for informing us of ref. [13].

\section{Appendix A}

CALCULATION OF THE ELECTROWEAK CONTRIBUTION TO THE MASS OF THE AXIONS

$$
M_{\imath j}^{2}=\left\langle a_{\mathrm{T}}^{t}\left|\delta \mathscr{L}_{\mathrm{EW}}\right| a_{\mathrm{T}}^{\prime}\right\rangle,
$$


where

$$
\begin{aligned}
\delta \mathscr{L}_{\mathrm{EW}}= & \frac{1}{2} \int \frac{\mathrm{d}^{4} q}{(2 \pi)^{4}} \frac{-g_{\mu \nu}}{\left(q^{2}-m_{\mathrm{W}}^{2}\right)} \int \mathrm{d}^{4} x \mathrm{e}^{\imath q x} \mathrm{~T}\left(J_{\mu}^{+}(x) J_{\nu}^{-}(0)\right) \\
& +\frac{1}{2} \int \frac{\mathrm{d}^{4} q}{(2 \pi)^{4}} \frac{-g^{\mu \nu}}{\left(q^{2}-m_{\mathrm{Z}}^{2}\right)} \int \mathrm{d}^{4} x \mathrm{e}^{\imath q x} \mathrm{~T}\left(J_{\mu}^{z}(x) J_{\nu}^{z}(0)\right) \\
& +\frac{1}{2} \int \frac{\mathrm{d}^{4} q}{(2 \pi)^{4}} \frac{-g^{\mu \nu}}{q^{2}} \int \mathrm{d}^{4} x \mathrm{e}^{\imath q x} \mathrm{~T}\left(J_{\mu}^{\gamma}(x) J_{\nu}^{\gamma}(0)\right)
\end{aligned}
$$

Using Dashen's formula we have

$$
M_{\imath}^{2}=\frac{1}{F_{\mathrm{T}}^{2}}\left\langle 0 \mid\left[Q_{5}^{\prime},\left[Q_{5}^{l}, \delta \mathscr{L}_{\mathrm{EW}}\right]\right] / 0\right\rangle
$$

where

$$
Q_{5}^{2} \equiv \int \mathrm{d}^{3} x J_{05}^{1}(x)
$$

and

$$
J_{\mu 5}^{\imath} \equiv \bar{\psi} \gamma_{\mu} \gamma_{5} \frac{\sigma^{\prime}}{4} \nabla_{\mathrm{T}} \psi,
$$

where
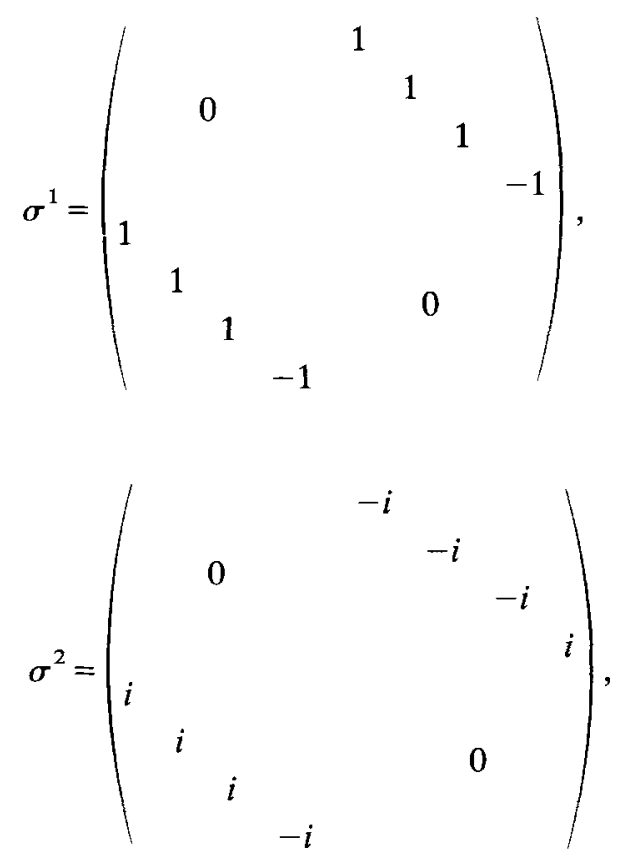
S. Dimopoulos et al. / Technicolor theories

$$
\sigma^{3}=\left(\begin{array}{cccccccc}
1 & & & & & & & \\
& 1 & & & & & & \\
& & 1 & & & & 0 & \\
& & & -1 & & & & \\
& & & & 1 & & & \\
& & & & & 1 & & \\
& 0 & & & & 1 & \\
& & & & & & -1
\end{array}\right) .
$$

In order to evaluate the commutator it is convenient to resort to a more general formalism. Consider thus

$$
M_{a b}^{2} \equiv\left\langle\pi_{\mathrm{T}}^{a}\left|\delta \mathscr{L}^{x}\right| \pi_{\mathrm{T}}^{b}\right\rangle,
$$

where the states $\pi_{\mathrm{T}}^{a}$ have been defined in eqs. (2.4) and (2.5) and

$$
\begin{gathered}
\delta \mathscr{L}^{x} \equiv \frac{1}{2} \int \frac{\mathrm{d}^{4} q}{(2 \pi)^{4}} \frac{-g^{\mu \nu}}{\left(q^{2}-m_{x}^{2}\right)} \int \mathrm{d}^{4} x \mathrm{e}^{i q x} \mathrm{~T}\left(J_{\mu}^{x}(x) J_{\nu}^{x}(0)\right), \\
J_{\mu}^{x} \equiv \bar{\psi} \gamma_{\mu} \Gamma x \psi,
\end{gathered}
$$

where $\Gamma_{x}$ is an $8 \times 8$ matrix (including the gauge coupling constant) and

$$
\Gamma_{\mathrm{x}} \equiv \Gamma_{\mathrm{V}}^{x}+\gamma_{5} \Gamma_{\mathrm{A}}^{x} \text {. }
$$

We then have

$$
M_{a b, x}^{2}=\frac{1}{F_{\mathrm{T}}^{2}}\left\langle 0\left|\left[Q_{5}^{b},\left[Q_{5}^{a}, \delta \mathscr{L}^{x}\right]\right]\right| 0\right\rangle
$$

where

$$
Q_{5}^{a} \equiv \int \mathrm{d}^{3} x J_{05}^{a}, \quad J_{\mu 5}^{a} \equiv \bar{\psi} \gamma_{\mu} \gamma_{5} t^{a} \psi
$$

[eq. (2.4)]. Upon evaluating the double commutator we obtain

$$
\begin{aligned}
M_{a b, x}^{2}= & \frac{1}{F_{\mathrm{T}}^{2}} \int \frac{\mathrm{d}^{4} q}{(2 \pi)^{4}} \frac{-1}{q^{2}-m_{x}^{2}} \int \mathrm{d}^{4} x \mathrm{e}^{\imath q x} \\
& \times\left\langle 0\left|\mathrm{~T}\left\{\begin{array}{l}
\psi \gamma_{\mu}\left[t^{b},\left[t^{a}, \Gamma_{x}\right]\right] \psi(x) \bar{\psi} \gamma^{\mu} \Gamma_{x} \psi(0) \\
+\bar{\psi} \gamma_{\mu} \gamma_{5}\left[t^{a}, \Gamma_{x}\right] \psi(x) \psi \gamma^{\mu} \gamma_{5}\left[t^{b}, \Gamma_{x}\right] \psi(0)
\end{array}\right\}\right| 0\right\rangle .
\end{aligned}
$$

Now following Das et al. [23] we define the vector (axial vector) spectral function $\rho_{\mathrm{V}}\left(\rho_{\mathrm{A}}\right)$ by the relation

$$
\int \mathrm{d}^{4} x \mathrm{e}^{\imath q x}\left\langle 0\left|\bar{\psi} \gamma_{\mu} t^{a} \psi(x) \bar{\psi} \gamma_{\nu} t^{b} \psi(0)\right| 0\right\rangle \equiv\left(q_{\mu} q_{\nu}-g_{\mu \nu} q^{2}\right) \delta_{\mathrm{ab}} \int \frac{\mathrm{d} m^{2} \rho_{\mathrm{V}}\left(m^{2}\right)}{q^{2}-m^{2}}
$$


and similarly for $\rho_{\mathrm{A}}$. Using the definition (A.9) and the algebraic relations of eq. (2.27) we obtain

$$
M_{a b, x}^{2}=\frac{3}{4 F_{\mathrm{F}}^{2} \pi^{2}} \int_{0}^{\infty} \frac{q^{4} \mathrm{~d} q^{2} \mathrm{~d} m^{2} \rho\left(m^{2}\right)}{\left(q^{2}+m_{x}^{2}\right)\left(q^{2}+m^{2}\right)} C_{a b, x},
$$

where we have made the standard Wick rotation into euclidean space; $\rho\left(m^{2}\right) \equiv$ $\rho_{\mathrm{A}}\left(m^{2}\right)-\rho_{\mathrm{V}}\left(m^{2}\right)$ and the Clebsch $C_{a b, x}$ is given by

$$
\begin{aligned}
C_{a b, x} \equiv & f_{a c d} f_{b c e}\left[\operatorname{Tr}\left(t^{d} \Gamma_{\mathrm{V}}^{x}\right) \operatorname{Tr}\left(t^{e} \Gamma_{\mathrm{V}}^{x}\right)\right. \\
& \left.-\operatorname{Tr}\left(t^{d} \Gamma_{\mathrm{A}}^{x}\right) \operatorname{Tr}\left(t^{e} \Gamma_{\mathrm{A}}^{x}\right)\right] .
\end{aligned}
$$

We note that the spectral function $\rho\left(\mathrm{m}^{2}\right)$ appears explicitly in the polarization tensor $\pi_{\mu \nu}\left(q^{2}\right)$ defined by

$$
\begin{aligned}
\delta_{a b} \pi_{\mu \nu}\left(q^{2}\right) & \equiv \int \mathrm{d}^{4} x \mathrm{e}^{l q x}\langle 0| \mathrm{T}\left(\bar{\psi} \gamma_{\mu}\left(1-\gamma_{5}\right) t^{a} \psi(x) \bar{\psi} \gamma_{\nu}\left(1+\gamma_{5}\right) t^{b} \psi(0)|0\rangle\right. \\
& =\left(q_{\mu} q_{\nu}-g_{\mu \nu} q^{2}\right) \int \frac{\mathrm{d} m^{2} \rho\left(m^{2}\right)}{q^{2}-m^{2}}
\end{aligned}
$$

With the use of the operator product expansion Bernard et al. [24] have shown that

$$
\delta_{a b} \pi_{\mu \nu}\left(q^{2}\right) \sim \sum_{i}\left\langle 0\left|\bar{\psi} \Gamma_{i} \psi \bar{\psi} \Gamma_{i} \psi\right| 0\right\rangle / q^{4}
$$

and thus we obtain the Weinberg sum rules:

$$
\begin{gathered}
\int \mathrm{d} m^{2} \rho\left(m^{2}\right)=0, \\
\int \mathrm{d} m^{2} m^{2} \rho\left(m^{2}\right)=0 .
\end{gathered}
$$

If we now saturate the Green function, eq. (A.12), with the narrow resonance $\pi_{\mathrm{T}}^{a}, \rho_{\mathrm{T}}^{a}, \mathrm{~A}_{\mathrm{T}}^{a}$ we have

$$
\rho\left(m^{2}\right)=F_{\mathrm{T}}^{2} \delta\left(m^{2}\right)+F_{\mathrm{A}_{\mathrm{T}}}^{2} \delta\left(m^{2}-m_{\mathrm{A}_{\mathrm{T}}}^{2}\right)-F_{\rho_{\mathrm{T}}}^{2} \delta\left(m^{2}-m_{\rho_{\mathrm{T}}}^{2}\right)
$$

or

$$
\begin{gathered}
F_{\mathrm{T}}^{2}+F_{\mathrm{A}_{\mathrm{T}}}^{2}=F_{\rho_{\mathrm{T}}}^{2}, \\
m_{\mathrm{A}_{\mathrm{T}} F_{\mathrm{A}_{\mathrm{T}}}^{2}}^{2}=m_{\rho_{\mathrm{T}}}^{2} F_{\rho_{\mathrm{T}}}^{2} .
\end{gathered}
$$

We may now evaluate the integral in eq. (A.10). We obtain

$$
\begin{aligned}
I & \equiv \int_{0}^{\infty} \mathrm{d} m^{2} \rho\left(m^{2}\right) \int_{0}^{\Lambda^{2}} \frac{q^{4} \mathrm{~d} q^{2}}{\left(q^{2}+m_{x}^{2}\right)\left(q^{2}+m^{2}\right)} \\
& \simeq \int_{0}^{\infty} \mathrm{d} m^{2} \rho\left(m^{2}\right) \frac{m_{x}^{4} \ln m_{x}^{2}-m^{4} \ln m^{2}}{m_{x}^{2}-m^{2}}
\end{aligned}
$$


where we have used the sum rules eq. (A.14). Now using eqs. (A.15) and (A.16) we find

$$
\begin{aligned}
I= & F_{\mathrm{T}}^{2}\left\{m_{x}^{2} \ln m_{x}^{2}+\frac{\varepsilon}{1-\varepsilon}\left(\frac{m_{x}^{4} \ln m_{x}^{2}-\left(m_{\rho_{\mathrm{T}}}^{4} / \varepsilon^{2}\right) \ln \left(m_{\rho_{\mathrm{T}}}^{2} / \varepsilon\right)}{m_{x}^{2}-m_{\rho_{\mathrm{T}}}^{2} / \varepsilon}\right)\right. \\
& \left.-\frac{1}{1-\varepsilon}\left(\frac{m_{x}^{4} \ln m_{x}^{2}-m_{\rho_{\mathrm{T}}}^{4} \ln m_{\rho_{\mathrm{T}}}^{2}}{m_{x}^{2}-m_{\rho_{\mathrm{T}}}^{2}}\right)\right\},
\end{aligned}
$$

where $\varepsilon \equiv m_{\rho_{\mathrm{T}}}^{2} / m_{\mathrm{A}_{\mathrm{T}}}^{2}$.

In the case of interest $x=\left\{\mathrm{W}^{ \pm}, \mathrm{Z}, \gamma\right\}$ and $m_{x} \ll m_{\rho_{\mathrm{T}}}$. In this limit we have

$$
I \sim F_{\mathrm{T}}^{2}\left\{\frac{m_{\rho_{\mathrm{T}}}^{2}}{1-\varepsilon} \ln \frac{1}{\varepsilon}+m_{x}^{2}\left(\ln \frac{m_{x}^{2}}{m_{\rho_{\mathrm{T}}}^{2}}+\frac{\varepsilon}{1-\varepsilon} \ln \frac{1}{\varepsilon}\right) .\right.
$$

Finally, combining eq. (A.19) with (A.10) we obtain

$$
M_{a b, x}^{2}=\frac{3}{4 F_{\mathrm{T}}^{2} \pi^{2}} I C_{a b, x} .
$$

Let us now evaluate the Clebsch $C_{a b, x}$ for $x=\left\{\mathrm{W}^{ \pm}, \mathrm{Z}, \gamma\right\}$. For the axions $t^{a} \equiv \frac{1}{4} \sigma^{t}, i=$ $1,2,3$, and the gauge currents eq. (A.6) are given by

$$
\begin{aligned}
\Gamma_{\mathrm{V}}^{\mathrm{W} \pm} & =g_{2}\left(\frac{\tau^{1} \pm i \tau^{2}}{2}\right)=-\Gamma_{\mathrm{A}}^{\mathrm{W}^{ \pm}}, \\
\Gamma_{\mathrm{V}}^{\mathrm{Z}} & =\frac{g_{2}}{\cos \theta_{\mathrm{W}}}\left(\frac{1}{4} \tau^{3}-\sin ^{2} \theta_{\mathrm{W}} Q\right), \\
\Gamma_{\mathrm{A}}^{\mathrm{Z}} & =\frac{-g_{2}}{\cos \theta_{\mathrm{W}}}\left(\frac{1}{4} \tau^{3}\right), \\
\Gamma_{\mathrm{V}}^{\gamma} & =g_{2} \sin \theta_{\mathrm{W}} Q, \\
\Gamma_{\mathrm{A}}^{\gamma} & \equiv 0,
\end{aligned}
$$

where

$$
Q \equiv\left(\begin{array}{cccccccc}
\frac{2}{3} & & & & & & & \\
& \frac{2}{3} & & & & & & \\
& & \frac{2}{3} & & & & & \\
& & 0 & & & & \\
& & & -\frac{1}{3} & & & \\
& & & & & -\frac{1}{3} & & \\
& & & & & & -\frac{1}{3} & \\
& & & & & & & -1
\end{array}\right) .
$$


We see immediately that the contribution of $\mathrm{W}^{ \pm}$to the axion mass vanishes. Thus we need only consider $Z^{0}$ and $\gamma$. Putting eq. (A.11) into the more tractable form

$$
C_{\imath,, x} \equiv \frac{1}{2}\left\{\operatorname{Tr}\left(\left[\frac{1}{4} \sigma^{2},\left[\frac{1}{4} \sigma^{\prime}, \Gamma_{\mathrm{V}}^{x}\right]\right] \Gamma_{\mathrm{V}}^{x}\right)-\operatorname{Tr}\left(\left[\frac{1}{4} \sigma^{l},\left[\frac{1}{4} \sigma^{J}, \Gamma_{\mathrm{A}}^{x}\right]\right] \Gamma_{\mathrm{A}}^{x}\right),\right.
$$

and using eq. (A.21) we obtain

$$
\begin{aligned}
& C_{33, \mathrm{z}} \equiv C_{33, \gamma} \equiv 0, \\
& C_{11, \mathrm{z}}=-\frac{1}{4} e^{2}=-C_{11, \gamma}, \\
& C_{22, \mathrm{z}}=-\frac{1}{4} e^{2}=-C_{22, \gamma},
\end{aligned}
$$

or finally

$$
\begin{aligned}
& M_{\mathrm{a}_{\mathrm{T}}^{3}}^{2}=0, \\
& M_{\mathrm{a}_{\mathrm{T}}}^{2}=\frac{3 \alpha}{4 \pi} m_{\mathrm{Z}_{\mathrm{o}}}^{2}\left(\ln \frac{m_{\rho_{\mathrm{T}}}^{2}}{m_{\mathrm{Z}_{\mathrm{O}}}^{2}}-\frac{\varepsilon}{1-\varepsilon} \ln \frac{1}{\varepsilon}\right),
\end{aligned}
$$

where

$$
\varepsilon \equiv m_{\rho_{\mathrm{T}}}^{2} / m_{\mathrm{A}_{\mathbf{T}}}^{2},
$$

Taking $\varepsilon=\frac{1}{2}$ and $m_{\rho_{\mathrm{T}}}=900 \mathrm{GeV}$ we find

$$
M_{\mathrm{a}_{\mathrm{T}}^{ \pm}}^{2}=(7.7 \mathrm{GeV})^{2} \text {. }
$$

\section{Appendix B}

In this appendix we estimate a lower bound on the mass of the Pati-Salam generator based on the fact that

$$
\frac{\Gamma\left(\mathrm{K}_{\mathrm{L}} \rightarrow \mu^{+} \mathrm{e}^{-}\right)}{\Gamma\left(\mathrm{K}^{+} \rightarrow \mu^{+} \nu\right)}<\left(2.5 \times 10^{-5}\right)^{2} .
$$

To do this we note that the Pati-Salam generators mediate the process $\mathrm{K}_{\mathrm{L}} \rightarrow \mu^{+} \mathrm{e}^{-}$ via the graph shown in fig 4 . The amplitude for this process is given by

$$
\begin{aligned}
\left\langle\mu^{+} \mathrm{e}^{-}\left|H_{\mathrm{I}}\right| \mathrm{K}_{\mathrm{L}}\right\rangle= & \left\langle\mu^{+} \mathrm{e}^{-}\left|\frac{g_{\mathrm{PS}}^{2}}{M_{p}^{2}} \bar{d} \gamma_{\mu} e \bar{\mu} \gamma_{\mu} s\right| \mathrm{K}_{\mathrm{L}}\right\rangle \\
= & \frac{g_{\mathrm{PS}}^{2}}{M_{P}^{2}}\left\{\frac{1}{2}\left\langle 0\left|\bar{d} \gamma_{\mu} \gamma_{S} s\right| \mathrm{K}_{\mathrm{L}}\right\rangle\left\langle\mu^{+} \mathrm{e}^{-}\left|\bar{\mu} \gamma_{\mu} \gamma_{5} e\right| 0\right\rangle\right\} \\
& -\left\langle 0\left|\bar{d} \gamma_{5} s\right| \mathrm{K}_{\mathrm{L}}\right\rangle\left\langle\mu^{+} \mathrm{e}^{-}\left|\bar{\mu} \gamma_{5} e\right| 0\right\rangle .
\end{aligned}
$$

The second equation is the result of a Fierz rotation. Now the matrix elements involving $\mathrm{K}_{\mathrm{L}}$ are estimated by using PCAC techniques as follows:

$$
\left\langle 0\left|\bar{d} \gamma_{\mu} \gamma_{5} s\right| \mathbf{K}_{\mathrm{L}}\right\rangle=i f_{\mathbf{K}} q_{\mu}
$$




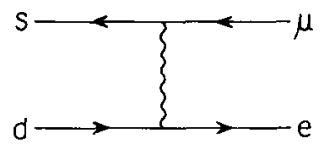

Fig. 4. The decay $\mathrm{K}^{0} \rightarrow \mu$ e mediated by a $t$-channel Pati-Salam boson

To estimate $\left\langle 0\left|\bar{d} \gamma_{5} s\right| \mathrm{K}_{\mathrm{L}}\right\rangle$ we use kaon pole dominance for the matrix element of the operator

$$
\begin{aligned}
\underset{q \rightarrow 0}{\mathrm{FT}} \partial_{\mu}\left\langle 0\left|\mathrm{~T}\left\{\bar{s} \gamma_{\mu} \gamma_{5} d(x) \bar{d} \gamma_{5} s(0)\right\}\right| 0\right\rangle=\left\langle 0\left|\left[Q_{5}^{\mathrm{K}^{\mathrm{0}}}, \bar{d} \gamma_{5} s\right]\right| 0\right\rangle \\
\quad=\langle 0|(\bar{s} s+\bar{d} d)| 0\rangle \\
\quad \simeq \underset{a \rightarrow 0}{\mathrm{FT}} \sum_{\imath=\mathrm{L}, s} \partial_{\mu}\left\langle 0\left|T\left\{j_{\mu 5}^{\mathrm{K}_{5}}\left|\mathrm{~K}_{\imath}\right\rangle\left\langle\mathrm{K}_{\imath}\right| \bar{d} \gamma_{5} s\right\}\right| 0\right\rangle \\
\quad=2\left\langle 0\left|\bar{d} \gamma_{5} s\right| \mathrm{K}_{\mathrm{L}}\right\rangle f_{\mathrm{K}} .
\end{aligned}
$$

These equations together with the standard relation for the kaon mass

$$
m_{\mathrm{K}}^{2} f_{\mathrm{K}}^{2}=\left(m_{\mathrm{s}}+m_{\mathrm{d}}\right)\langle 0|\bar{q} q| 0\rangle,
$$

result in the following form for $\mathrm{K}_{\mathrm{L}} \rightarrow \mu^{+} e^{-}$:

$$
\left\langle\mu^{+} \mathrm{e}^{-}\left|H_{I}\right| \mathrm{K}_{\mathrm{L}}\right\rangle=\frac{g_{\mathrm{PS}}^{2}}{M_{p}^{2}}\left\{\frac{1}{2} f_{\mathrm{K}}\left(m_{\mu}+m_{e}\right)-\frac{m_{\mathrm{K}}^{2} \circ f_{\mathrm{K}}}{m_{\mathrm{s}}+m_{\mathrm{d}}}\right\} \bar{\mu} \gamma_{5} e
$$

The second (pseudoscalar) term clearly dominates. Thus the rate for $\mathrm{K}_{\mathrm{L}} \rightarrow \mu^{+} \mathrm{e}^{-}$is given by

$$
\Gamma\left(\mathrm{K}_{\mathrm{L}} \rightarrow \mu^{+} \mathrm{e}^{-}\right) \simeq\left\{\frac{g_{\mathrm{PS}}^{2}}{M_{p}^{2}} \frac{m_{\mathrm{K}^{0} f_{\mathrm{K}}}^{2}}{\left(m_{\mathrm{s}}+m_{\mathrm{d}}\right)}\right\}^{2} 2 m_{\mathrm{K}}^{2} \times\left\{\begin{array}{c}
\mu^{+} \mathrm{e}^{-} \\
\text {phase space }
\end{array}\right\}
$$

This is to be compared to the standard rate

$$
\Gamma\left(\mathrm{K}^{+} \rightarrow \mu^{+} \nu\right) \simeq \frac{1}{2} f_{\mathrm{K}}^{2} m_{\mathrm{K}}^{2}\left\{\frac{g_{2}^{2}}{M_{\mathrm{W}}^{2}} \sin \theta_{\mathrm{C}}\right\}^{2} \times\left\{\begin{array}{c}
\mu^{+} \nu \\
\text { phase space }
\end{array}\right\} .
$$

Dividing (B.7) by (B.8) and using (B.1), we obtain

$$
\frac{\Gamma\left(\mathrm{K}_{\mathrm{L}} \rightarrow \mu^{+} \mathrm{e}^{-}\right)}{\Gamma\left(\mathrm{K}^{+} \rightarrow \mu^{+} \nu\right)} \simeq\left\{\frac{g_{\mathrm{PS}}^{2}}{M_{p}^{2}} \frac{M_{\mathrm{W}}^{2}}{g_{2}^{2} \sin \theta_{\mathrm{C}}}\right\}^{2} 4\left\{\frac{m_{\mathrm{K}}^{2}}{m_{\mu} m_{\mathrm{s}}}\right\}^{2}<\left(2.5 \times 10^{-5}\right)^{2}
$$

which finally yields

$$
\left(\frac{M_{p}}{g_{\mathrm{PS}}}\right)>310 \mathrm{TeV}
$$




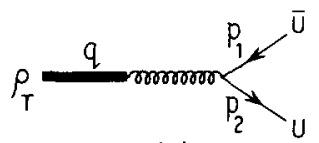

(a)

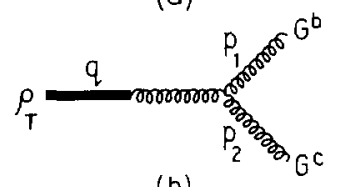

(b)

Fig. 5. Production mechanisms for $\rho_{\mathrm{T}}^{a}$.

\section{Appendix C}

CALCULATION OF THE DECAY RATES OF EQ. (4 8) [11]

In order to calculate the decay rate of a flavor singlet, color octet technirho we use standard vector meson dominance ideas. Let $J_{\mu}^{a}$ be the gauge current which acts as a source for $\rho_{\mathrm{T}}^{a}$. In general we have

$$
J_{\mu}^{a} \equiv \bar{\psi} \gamma_{\mu} t^{a} \psi
$$

[where $t^{a}$ are defined in eq. (2.4)] and in addition

$$
\left\langle 0\left|J_{\mu}^{a}\right| \rho_{\mathrm{T}}^{b}\right\rangle \equiv i F_{\rho_{\mathrm{T}}} m_{\rho} \varepsilon_{\mu} \delta_{a b} .
$$

The decay amplitude for the processes $\rho_{\mathrm{T}}^{a} \rightarrow \overline{\mathrm{u} u}, \overline{\mathrm{d} d}$, GG (see figs. 5a, b) are as follows:

$$
\begin{aligned}
T_{\rho_{\mathrm{T}} \mathrm{a} \rightarrow \overline{\mathrm{u}} \mathrm{u}} & =\bar{u} \frac{1}{2} \lambda_{a} \gamma_{\mu} u \frac{g_{3}^{2}}{m_{\rho_{\mathrm{T}}}^{2}} \sqrt{2} M_{\rho_{\mathrm{T}}} F_{\rho_{\mathrm{T}}} \varepsilon^{\mu}(q), \\
T_{\rho_{\mathrm{T}}^{a} \rightarrow \overline{\mathrm{d} d}} & =\bar{d} \frac{1}{2} \lambda_{a} \gamma_{\mu} d \frac{g_{3}^{2}}{m_{\rho_{\mathrm{T}}}^{2}} \sqrt{2} m_{\rho_{\mathrm{T}}} F_{\rho_{\mathrm{T}}} \varepsilon^{\mu}(q), \\
T_{\rho_{\mathrm{T} \rightarrow \mathrm{GG}}^{a}} & =\varepsilon^{\star}\left(p_{1}\right) \varepsilon^{\star}\left(p_{2}\right)\left(p_{1}-p_{2}\right)_{\mu} f_{a b c} \frac{g_{3}^{2}}{m_{\rho_{\mathrm{T}}}^{2}} \sqrt{2} M_{\rho_{\mathrm{T}}} F_{\rho_{\mathrm{T}}} \varepsilon^{\mu}(q) .
\end{aligned}
$$

We then obtain the decay rates

$$
\begin{aligned}
& \Gamma_{\rho \mathrm{T} \rightarrow \overline{\mathrm{u}} \mathrm{u}}^{\mathrm{a}} \equiv \Gamma_{\rho_{\mathrm{T}} \rightarrow \overline{\mathrm{d} d}}=\frac{4 \pi}{3} \alpha_{3}^{2} \frac{F_{\rho_{\mathrm{T}}}^{2}}{m_{\rho \mathrm{T}}}, \\
& \Gamma_{\rho_{\mathrm{T}}^{\mathrm{a}} \rightarrow \mathrm{GG}} \equiv 2 \pi \alpha_{3}^{2} \frac{F_{\rho_{\mathrm{T}}}^{2}}{M_{\rho_{\mathrm{T}}}} .
\end{aligned}
$$

\section{References}

[1] S Weinberg, Phys. Rev Lett. 19 (1967) 1264,

A. Salam, in Elementary particle physics, ed N. Svartholm (Almqvist and Wiksell, Stockholm, 1968) 
[2] L. Susskınd, SLAC preprint (May, 1978);

S Weinberg, Phys Rev. D19 (1978) 1277

[3] S Dimopoulos and L. Susskınd, Nucl. Phys. B155 (1979) 237

[4] E. Eichten and K. Lane, Harvard preprint HUTP 79/A002 (1979)

[5] E Fahrı and L Susskind, SLAC Pub. 2361 (1979)

[6] M. Gell-Mann, P. Ramond and R. Slansky, in preparation

[7] S Dimopoulos, S Raby and P. Sikıvie, Nucl. Phys B176 (1980) 449

[8] S. Dimopoulos, Nucl. Phys. B168 (1980) 93

[9] S Dimopoulos, S Raby and L Susskınd, Technicolor, Talk at McGıll APS Conf. Nov., 1979

[10] M.A.B Beg, D Politzer and P. Ramond, Phys. Rev Lett 43 (1979) 1701

[11] J Bjorken, unpublished

[12] M. Peskın, Saclay preprınt (May, 1980)

[13] J. Preskill, Nucl. Phys B177 (1981) 21

[14] G.L Kane, Talk at Madison Conf. on Production of new partıcles, Oct., 1979

[15] G. 't Hooft, Nucl Phys. B72 (1974) 461

[16] R D. Pecceı, Review of axıons, Proc 19th Int. Conf on High-energy physics, Tokyo, 1978

[17] R. Dashen, Phys. Rev 183 (1969) 1245

[18] M.A. Shifman, A I. Vainsteın, V.I. Zakharov, Nucl Phys B147 (1979) 385, 448, 519

[19] S. Adler, Brandess Summer School lectures, 1970

[20] P Sikıvıe, L Susskind, M. Voloshın and V. Zakharov, Nucl Phys. B173 (1980) 189

[21] H Georgi, S. Glashow, M. Machacek and D Nanopoulos, Phys. Rev. Lett. 40 (1978) 692

[22] C Albright, J Smith and H Tye, Phys. Rev. 21 (1980) 711

[23] T Das et al, Phys Rev Lett 18 (1967) 759

[24] C Bernard et al, Phys Rev D12 (1975) 792 